\title{
The long term outcome of gastric non-invasive neoplasia
}

\author{
M Rugge, M Cassaro, F Di Mario, G Leo, G Leandro, V M Russo, G Pennelli, F Farinati \\ for the Interdisciplinary Group on Gastric Epithelial Dysplasia (IGGED)
}

See end of article for authors' affiliations

Correspondence to:

Dr M Rugge, III Cattedra di

Anatomia Patologica,

Università degli Studi di

Padova, Via Aristide

Gabelli, 61, 35121,

Padova, Italia;

massimo.rugge@.unipd.it

Accepted for publication: 3 March 2003
Background: The cancer risk associated with gastric non-invasive neoplasia (formerly dysplasia) is debated. This prospective long term follow up study investigates the clinicopathological behaviour of non-invasive gastric neoplasia (and related lesions), focusing on the cancer risk associated with each different histological phenotype.

Patients and methods: A total of 118 consecutive cases (nine indefinite for non- invasive neoplasia; 90 low grade non-invasive neoplasia; 16 high grade non- invasive neoplasia; and three suspicious for invasive adenocarcinoma) with a histological follow up of more than 12 months (average 52 months; range 12-206) were prospectively followed up with a standardised protocol. Patients in whom gastric cancer was detected within 12 months from the initial diagnosis of non-invasive neoplasia were excluded, assuming that invasive carcinoma had been missed at the initial endoscopy procedure. Results: Non-invasive neoplasia was no longer detectable in $57 / 118$ cases (48\%), was unchanged in $32(30 \%)$, and evolved into gastric cancer in 20 patients (17\%). Evolution to invasive adenocarcinoma was documented in both low and high grade non-invasive neoplastic lesions (8/90 low grade; $11 / 16$ high grade) and correlated with histological severity (low versus high grade) at baseline $(p<0.001)$. Seventy five per cent of cancers occurring during the long term follow up were stage I. Conclusions: The risk of invasive gastric cancer increases with the histological grade of the non-invasive neoplasia. Following up non-invasive gastric neoplasia increases the likelihood of gastric cancer being detected in its early stages. nvasive cancer is the final step in a cascade of morphological changes which have been defined as multistep oncogenesis. ${ }^{2}$ This process includes a continuum of progressively dedifferentiated phenotypes, which may result

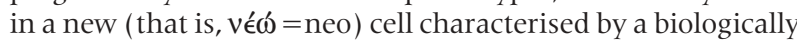
deregulated, potentially metastatic growth (that is, $\pi \lambda \alpha \zeta \omega=$ plasia $)$. According to the definition of the World Health Organization, ${ }^{3}$ non-invasive gastric neoplasia (formerly dysplasia ${ }^{3-8}$ ) indicates a "pre-invasive neoplastic change" of the gastric glands, and therefore its clinicopathological characterisation has important implications for both primary and secondary gastric cancer (GC) prevention.

Several different terms have been used for non-invasive neoplasia arising in the gastric mucosa. Irrespective of the terminology used, numerous studies have revealed the existence of a remarkable interobserver variation in the histological assessment of non-invasive neoplasia. ${ }^{4-14}$ The whole histological spectrum of gastric precancerous lesions has recently been revisited and the diagnostic criteria extensively described. ${ }^{15}$ Tested among members of an international group of specialised pathologists, the proposed classification has demonstrated a significant interobserver diagnostic consistency. ${ }^{15}$

This long term, prospective, follow up study aimed to describe the pathological outcome of histological lesions belonging to the spectrum of gastric non-invasive neoplasia, also focusing on the relationship between such morphological alterations and the onset of invasive cancer.

\section{PATIENTS AND METHODS}

\section{Patients}

In 1985-1986, an Interdisciplinary Group was established by various centres in the northeast of Italy to perform a prospective study on the clinicopathological outcome of gastric non-invasive neoplasia. ${ }^{16-18}$ Any patient in whom non-invasive neoplasia was histologically diagnosed at gastric endoscopy was asked to join a follow up protocol, based on the histological typing of the lesion. In accordance with the aims of the study, patients who had previously undergone surgical or endoscopic treatments for gastric neoplasms were ruled out.

Focusing on the long term follow up of non-invasive neoplastic lesions, the present report did not consider patients with a follow up of less than 12 months - that is, (i) patients who failed to comply with the follow up protocol; (ii) patients who were diagnosed as having stomach cancer within 12 months of the initial diagnosis of non-invasive neoplasia (or related lesions); and (iii) patients enrolled in the study (which is still underway) who have been followed up for less than a year.

Figure 1 shows the timing of the endoscopy procedures. From early 1987 to January 2001, a total of 161 consecutive patients were enrolled in the prospective study. This study only reports the clinicopathological outcome of 118 subjects with a follow up of more than 12 months (mean 52; range 12-206): 67 were male (mean age 55 years, range 29-83) and 51 were female (mean age 58 years, range 32-79) (table 1). At the baseline endoscopy procedure, 72 patients had non-ulcer dyspepsia, while active gastric or duodenal ulcer was detected in 19/118 and 13/118 patients, respectively. Active peptic ulcer was detected in one patient at both the gastric and duodenal levels (table 1). The original clinical records did not report the baseline endoscopy findings in the remaining 13 cases (11\%). After joining the study, even in the absence of any endoscopy abnormalities, all patients underwent standardised biopsy sampling of the gastric mucosa (fig 2). Additional samples were always taken from any focal lesions (ulcer, mucosal flat abnormalities, and polyps). All patients had more than three endoscopic check ups (mean procedures per patient $=9.1$ ). During the prospective study, no patient had endoscopic mucosal resections (EMR) other than polypectomy (performed in one case of high grade non-invasive neoplasia

Abbreviations: GC, gastric cancer; EGC, early gastric cancer; AGC advanced gastric cancer; EMR, endoscopic mucosal resections; GC-nos, gastric cancer not otherwise specified. 


\begin{tabular}{|lllll|}
\hline & \multicolumn{5}{c|}{ Follow up schedule } \\
(months from the initial diagnosis)
\end{tabular}

Figure 1 Follow up protocol. Lesions indefinite for non-invasive neoplasia were followed up as scheduled until the case was assigned to the non-neoplastic or neoplastic non-invasive category. Patients were considered disease free after two successive endoscopy/biopsy procedures consistently negative for non-invasive neoplasia. When the grade of the lesion was consistently lower than before in two successive tests (that is, "regression" from high grade to low grade), the patient was followed up in accordance with the recommendations for low grade non-invasive neoplasia. High grade lesions detected during the follow up of low grade non-invasive neoplasia were subsequently followed up as high grade lesions.

detected within a polypoid lesion completely removed at endoscopy).

For ethical reasons, patients with two consecutive endoscopy procedures (both with extensive biopsy samplings and with a follow up never shorter than 12 months) consistently failing to detect non-invasive neoplasia were not asked to submit to further follow up; such cases were conventionally described as having regressed (see the conventional terminology extensively explained below).

In the absence of evidence based guidelines on the management of high grade non- invasive gastric neoplasia, each centre involved in the study was allowed to decide on the management of patients with high grade lesions.

\section{Histological assessment}

By definition, the histological diagnosis of non-invasive neoplasia was restricted to cases showing both altered glandular architecture and abnormalities in cytology and differentiation, but lacking any (even doubtful) infiltrating features. ${ }^{35}$ The histological classification was based on serial sections of each biopsy sample (haematoxylin-eosin stain). The presence of Helicobacter pylori was ascertained microscopically (by modified Giemsa stain and/or immunostain; Dako monoclonal antibodies, Milan, Italy). According to the classification adopted, the diagnosis of "indefinite for non-invasive neoplasia" was appropriate when it was impossible to classify cases as either unequivocally negative or positive for non-invasive neoplasia. ${ }^{315} 19$

To minimise interobserver variation, all biopsies were evaluated by the same coordinating pathologist (MR). Tested in a random sample of 50 biopsies, the intraobserver diagnostic consistency, calculated by $K$ statistics, was 0.78 .

\section{Conventional definitions of non-invasive neoplasia outcome}

Excluding any reference to the biological behaviour of the lesion, a conventional nomenclature was used to describe the clinicopathological outcome of non-invasive neoplasia.

Regression. In at least two subsequent tests, non-invasive neoplasia was either no longer detected or always found to a lesser degree. Even in the context of an extensive sampling protocol, repeated sampling defects cannot be ruled out entirely however. Indeed, this remains a structural limit of the present study, in common with other endoscopic follow up studies dealing with gastric precancerous lesions. In fact, such lesions generally lack the polypoid appearance that represents the most suitable marker of precancerous changes in the large bowel district. In addition, the likelihood of small foci of noninvasive neoplasia being removed at the initial biopsy cannot be entirely ruled out. To minimise the risk of focal abnormalities being overlooked during the follow up, the definition of "regression" of low grade non-invasive neoplasia strictly required both: (i) at least two "negative" endoscopic check ups and (ii) a follow up of at least 12 months after the first negative biopsy test. Regression from high grade to low grade noninvasive neoplasia (or to absence of non-invasive neoplasia) was defined consistently.

Persistence. No changes in the grade of non-invasive neoplasia assessed in two subsequent tests.

Progression. High grade non-invasive neoplasia (following a previous finding of a low grade lesion) or GC detected during follow up. Non-invasive neoplasia (both high and low grade) was assumed to evolve into invasive cancer when GC was histologically demonstrated no less than 12 months after the initial diagnosis of non-invasive neoplasia.

\section{Gastric (invasive) carcinoma (GC) detected during follow up}

This long term, prospective, follow up study only considered patients followed up for non-invasive neoplasia (or related lesions) for a period of more than 12 months. Consequently, patients were excluded whose invasive cancer was demonstrated within 12 months of the initial diagnosis of non-invasive neoplasia (or related lesions) ${ }^{20}$ In designing this study, such "early detected GCs" were assumed to have been missed at the initial endoscopy and were regarded as non-informative on the long term clinicopathological outcome of the histological lesions considered in the study. Gastric adenocarcinoma detected during this long term follow up was distinguished as early (EGC) and advanced (AGC) on the basis of the pathological stage ascertained after surgery. ${ }^{21}$ Histologically confirmed GC in patients who did not undergo surgery was defined as not otherwise specified (GC-nos).

\section{Statistical methods}

Frequency tables were analysed using Yates' corrected $\chi^{2}$ and Pearson's $\chi^{2}$. The test for linear trend was used when appropriate. Clinicopathological outcome of non-invasive neoplasia (that is, low grade $v$ high grade) was analysed using Life Tables (Kaplan-Meier method). The groups were compared using the Mantel-Cox test. Bio-Medical Data Processing software (BMDP; University of California, USA) was used for all calculations. A p value $<0.05$ was considered significant.

\section{RESULTS}

Outcome of cases indefinite for non-invasive neoplasia Among the nine patients (7.6\%) enrolled with lesions indefinite for non-invasive neoplasia, follow up failed to detect any neoplastic non-invasive lesions in eight cases. In one patient, who was $H$ pylori positive, check up endoscopy demonstrated low grade non-invasive neoplasia. After the diagnosis of low grade non-invasive neoplasia was established, this patient underwent $H$ pylori eradication and was followed up for more than three years during which time no change occurred in the grade of the lesion. One patient with lesions indefinite for non-invasive neoplasia that were not associated with $H$ pylori infection or intestinal metaplasia had a clinical history of non-steroidal anti-inflammatory drug intake and cytohistological atypia coexisting with microerosive gastritis. After two negative endoscopies/biopsies, the lesion was considered regressed. A new endoscopy check up, performed after more than two years, did not reveal histology lesions other than minimal interfoveolar inflammation (antral specimens).

\section{Non-invasive neoplasia: histological grades, $\boldsymbol{H}$ pylori} status, and overall outcome

At the initial diagnosis, non-invasive neoplasia was judged to be low grade in 90 of $118(76 \%)$ patients and high grade in 16 ( $14 \%)$. In three patients (2.5\%), the initial diagnosis was high 
Table 1 Demographic data on all patients with a follow up longer than 12 months. For each diagnostic category, mean follow up, patient characteristics at the initial endoscopy procedure, and Helicobacter pylori (Hp) status at enrolment are also shown

\begin{tabular}{|c|c|c|c|c|c|c|}
\hline Diagnostic category & $\begin{array}{l}\text { No of } \\
\text { patients } \\
\text { (M/F) }\end{array}$ & Age (y) & $\begin{array}{l}\text { Follow up } \\
\text { (months) }\end{array}$ & $\begin{array}{l}\text { Characteristics of patients (baseline } \\
\text { endoscopy procedure) }\end{array}$ & & $\begin{array}{l}\text { Hp positive/Hp } \\
\text { negative at enrolment }\end{array}$ \\
\hline \multirow[t]{5}{*}{ Indefinite for non-invasive neoplasia } & $9(7 / 2)$ & $48(34-71)$ & $42(24-94)$ & Non-ulcer dyspepsia & 5 & $8 / 1$ \\
\hline & & & & Gastric ulcer (active) & 1 & \\
\hline & & & & Duodenal ulcer (active) & 2 & \\
\hline & & & & Gastric+duodenal ulcer (active) & 0 & \\
\hline & & & & Unknown & 1 & \\
\hline \multirow[t]{5}{*}{ Low grade non-invasive neoplasia } & $90(48 / 42)$ & $56(31-83)$ & $52(12-206)$ & Non-ulcer dyspepsia & 57 & $86 / 4$ \\
\hline & & & & Gastric ulcer (active) & 12 & \\
\hline & & & & Duodenal ulcer (active) & 10 & \\
\hline & & & & Gastric+duodenal ulcer (active) & 1 & \\
\hline & & & & Unknown & 10 & \\
\hline \multirow{5}{*}{ High grade non-invasive neoplasia } & $16(10 / 6)$ & $60(29-79)$ & $34(12-72)$ & Non-ulcer dyspepsia & 9 & $14 / 2$ \\
\hline & & & & Gastric ulcer (active) & 5 & \\
\hline & & & & Duodenal ulcer (active) & 1 & \\
\hline & & & & Gastric+duodenal ulcer & 0 & \\
\hline & & & & Unknown & 1 & \\
\hline \multirow[t]{5}{*}{ Suspicious for invasive cancer } & $3(2 / 1)$ & $65(60-70)$ & $19(12-30)$ & Non-ulcer dyspepsia & 1 & $3 / 0$ \\
\hline & & & & Gastric ulcer (active) & 1 & \\
\hline & & & & Duodenal ulcer (active) & 0 & \\
\hline & & & & Gastric+duodenal ulcer (active) & 0 & \\
\hline & & & & Unknown & 1 & \\
\hline \multirow[t]{5}{*}{ Total } & $118(67 / 51)$ & $56(29-83)$ & $52(12-206)$ & Non-ulcer dyspepsia & 72 & $111 / 7$ \\
\hline & & & & Gastric ulcer (active) & 19 & \\
\hline & & & & Duodenal ulcer (active) & 13 & \\
\hline & & & & Gastric+duodenal ulcer (active) & 1 & \\
\hline & & & & Unknown & 13 & \\
\hline
\end{tabular}

grade non-invasive neoplasia coexisting with areas of suspected invasive adenocarcinoma. For each diagnostic category, table 1 shows the demographic data, median follow up, and $H$ pylori status at enrolment. At the initial diagnosis, 111/118 (93.2\%) patients were $H$ pylori positive. In seven cases (one indefinite for non-invasive neoplasia, four low grade, and two high grade non-invasive neoplasia), histology failed to reveal $H$ pylori infection at the initial or follow up biopsy. None of these patients had ever received anti-H pylori therapy and all but one showed extensive intestinalisation of the gastric mucosa. The overall outcome for non-invasive neoplastic lesions is shown in table 2. A significant association was established between the grade of non-invasive neoplasia at

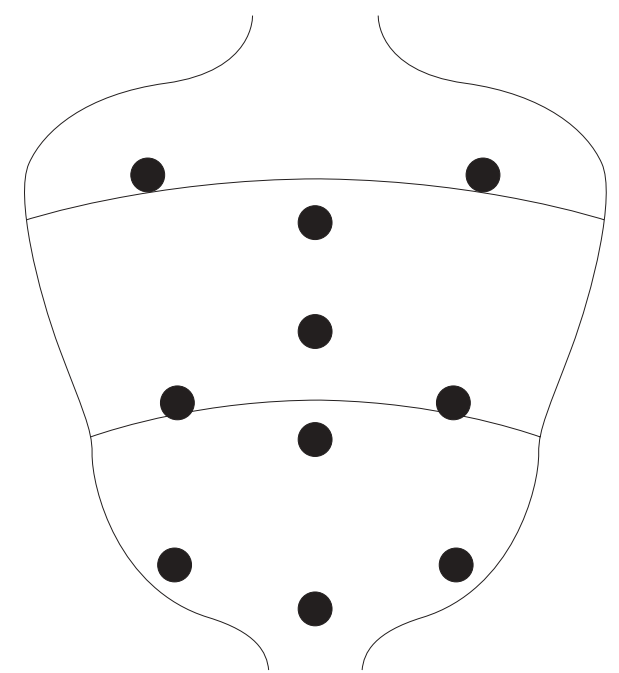

Figure 2 Gastric biopsy sampling protocol (dots show sites of biopsy). During follow up, after an initial diagnosis of non-invasive neoplasia, all patients underwent endoscopy with multiple biopsy sampling; 1-3 samples were obtained from each site. The sampling protocol demands further sampling of any focal lesions. baseline and its clinicopathological outcome-that is, the higher the grade, the higher the risk of progression and evolution into GC (test for linear trend: $\mathrm{p}<0.001$ ).

\section{Outcome of low grade non-invasive neoplasia}

Low grade non-invasive neoplasia regressed in 48/90 patients $(53.3 \%)$ who were not asked to undergo any further follow up. Persistent low grade non-invasive neoplasia was recorded in 28 cases $(31.1 \%)$ and progression to the higher grade was observed in six patients $(6.6 \%)$. In eight cases $(8.8 \%)$, low grade non-invasive lesions evolved into GC after a mean 48 months of follow up (range 21-85).

\section{Outcome of high grade non-invasive neoplasia}

During the long term follow up (mean 34 months; range 13-72), all but five cases of high grade lesions progressed to GC. Among the five cases that did not evolve into GC, four are still being followed up for high grade lesions while noninvasive neoplasia was no longer detected in one $(6.2 \%)$; in this case of "regression", the high grade lesion lay within a pedunculated polyp, which was completely excised by endoscopy. After polypectomy, repeated endoscopies failed to detect any non-invasive neoplastic lesions in the context of atrophicmetaplastic gastritis. ${ }^{22}$

The risk of developing into GC was significantly higher for high grade non-invasive neoplasia than for low grade lesions $(\mathrm{p}<0.001)$ (fig 3)

\section{Outcome of high grade non-invasive neoplasia coexisting with suspected areas of invasion}

At enrolment, three cases were classified as high grade non-invasive neoplasia coexisting with suspected areas of invasive cancer. During the subsequent follow up, two of three cases were classified as high grade non-invasive neoplasia while an invasive GC was histologically demonstrated in one case.

Invasive adenocarcinoma in the long term follow up of non-invasive neoplasia

Distinguishing early (EGC) from advanced (AGC) and not otherwise specified GCs (GC- nos), table 3 shows the evolution 
Table 2 Clinicopathological outcome of non-invasive neoplasia

\begin{tabular}{lccccc}
\hline & \multicolumn{2}{l}{ Final diagnosis } & \\
\cline { 2 - 4 } & \multicolumn{2}{l}{ Non-invasive neoplasia } & \\
\cline { 2 - 4 } Initial diagnosis & Absent & Low grade & High grade & Invasive cancer & Total \\
\hline Indefinite for non-invasive neoplasia & 8 & 1 & 0 & 0 & 9 \\
Low grade non-invasive neoplasia & 48 & 28 & 6 & 8 & 90 \\
High grade non-invasive neoplasia & 1 & 0 & 4 & 11 & 16 \\
Suspicious for invasive cancer & 0 & 0 & 2 & 1 & 3 \\
\hline
\end{tabular}

Initial diagnosis: histological diagnosis at enrolment; final diagnosis: diagnosis at end of follow up; absent: no non-invasive neoplasia detected.

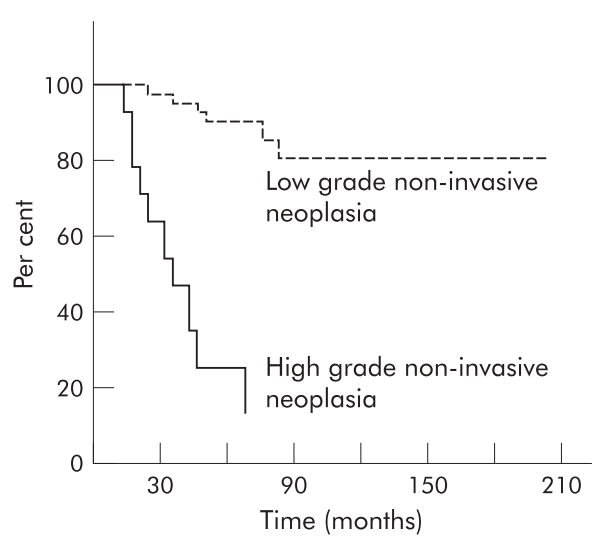

Figure 3 Low grade versus high grade gastric non-invasive neoplasia: evolution into invasive adenocarcinoma $(p<0.001)$.

of gastric non-invasive neoplasia into invasive adenocarcinoma. Among the 20 cases of invasive cancer, 13 were pathologically staged as EGC (86.6\%) while only two were staged as advanced (table 3 ).

\section{DISCUSSION}

In countries other than Japan, the vast majority of GCs diagnosed are already in an advanced stage, with a five year survival rate lower than $20 \%{ }^{23}{ }^{24}$ In Japan, approximately half of GCs are detected at an early stage and surgical treatment results in 10 year survival rates higher than $85 \% .^{25}$ The Japanese experience demonstrates that the early detection of GC dramatically improves its prognosis, and therefore it is worth asking whether there are potential clinical benefits in following up (and/or treating) high risk gastric precancerous lesions and non-invasive neoplasia in particular. ${ }^{26-30}$

On the whole, this prospective study demonstrates that, during long term follow up, the clinicopathological outcome of a non-invasive gastric neoplasia correlates significantly with the grade of the lesion at presentation.

\section{Indefinite for non-invasive neoplasia}

The present results support both the biological heterogeneity of the phenotype "indefinite for non-invasive neoplasia" and the clinical usefulness of such a histological classification. ${ }^{15} 19$ The indefinite for non-invasive neoplasia phenotype occurs most frequently in association with $H$ pylori infection. In such cases, it might be speculated that any eradication therapy, by lowering the meta-inflammatory component of the regenerative epithelial changes, could result in a more suitable categorisation of the mucosal lesions. In the present series, in the only case of alterations indefinite for non-invasive neoplasia occurring in a $H$ pylori negative patient, the lesions were no longer detected after stopping the patient's non-steroidal antiinflammatory drug intake.

\section{Outcome of low grade non-invasive neoplasia}

Low grade non-invasive neoplasia was the most prevalent $(76 \%)$ of the non-invasive neoplastic lesions, although difficulties encountered in distinguishing low grade from regenerative (that is, inflammatory) lesions may result in an excessive use of the former diagnostic category. ${ }^{30}{ }^{31}$ Even when well codified diagnostic criteria are applied, the results of this study support the biological heterogeneity of the histological phenotype of low grade non-invasive neoplasia. In fact, while most low grade lesions were not confirmed at subsequent check ups, a minority persisted or progressed to high grade lesions or GC during a follow up of more than two years. Eight cases of evolution into adenocarcinoma were observed (with a mean follow up longer than three years), which supports the cost effectiveness of a follow up strategy in all cases of low grade non-invasive neoplasia.

\section{Outcome of high grade non-invasive neoplasia}

High grade non-invasive neoplasia requires both high grade cytoarchitectural atypia and the absence of any histological evidence of stromal invasion. Data provided in the literature are difficult to compare with the findings of the present study

\begin{tabular}{|c|c|c|c|c|c|}
\hline & $\begin{array}{l}\text { Follow up } \\
\text { (months) }\end{array}$ & EGC & AGC & GC-nos & $\begin{array}{l}\text { GCs developing } \\
\text { during follow up }\end{array}$ \\
\hline Low grade non-invasive neoplasia & $48(21-85)$ & 5 & 1 & 2 & 8 \\
\hline High grade non-invasive neoplasia & $30(13-72)$ & 7 & 1 & 3 & 11 \\
\hline Suspicious for invasive cancer & 30 & 1 & 0 & 0 & 1 \\
\hline Total & $37(13-85)$ & 13 & 2 & 5 & 20 \\
\hline \multicolumn{6}{|c|}{$\begin{array}{l}\text { *Values are mean (range). Duration of follow up is the time that elapsed between the initial endoscopy } \\
\text { procedure and the histological diagnosis of gastric cancer. } \\
\text { EGC, early gastric cancer (that is, pathological stage I); AGC, advanced gastric cancer (that is, pathological } \\
\text { stages II-III); GC-nos, gastric cancer of unknown pathological stage. }\end{array}$} \\
\hline
\end{tabular}


for two reasons: (1) in grading gastric non-invasive neoplasia, most of the published studies adopted a three tiered scale (that is, mild, moderate, severe); (2) to the best of our knowledge, no such prospective long term follow up studies have been published.

The prevalence of the invasive evolution of moderate dysplasia has been shown to be lower than $10 \%,{ }^{32-35}$ with the significant exception reported by Fertitta et al, who recorded a progression to GC in 7 of 21 cases. $^{36}$

In previously published studies, regression of moderate dysplasia ranged between $27 \%$ and $60 \%$ of cases, ${ }^{36}{ }^{37}$ but these studies provide incomplete follow up information on patients whose dysplastic lesion was subsequently no longer detectable. Recently, Kokkola et al reported on eight cases $(57 \%)$ of regression in a series of 14 patients enrolled with moderate dysplasia. ${ }^{38}$ It is worth noting however that in three cases this regression was due to the endoscopic resection of the polyps in which the dysplasia had been identified.

Data in the literature demonstrate that, in the majority of cases, severe dysplasia either coexists with or is a precursor of GC. ${ }^{17535-42}$ This would justify the ablation of lesions by traditional surgery or EMR. ${ }^{26}$

In our experience, high grade non-invasive neoplasia never regressed (except for the case in which the lesion was located in a pedunculated polyp, which had been completely removed at endoscopy) and we consider this as convincing evidence to support surgical treatment (or EMR where possible and/or available) for all high grade non-invasive neoplastic lesions.

\section{Non-invasive neoplasia and invasive GC}

All 118 patients enrolled in our study were followed up for a period ranging from 12 to 206 months (mean 52 months), during which time 20 GCs were detected. The time elapsing between enrolment and diagnosis of invasive adenocarcinoma in these case ranged between 17 and 80 months, a period consistent with the biological evolution from a non- invasive to an invasive neoplasm. In 13/20 cases (65\%), the pathological stage ascertained after surgery was stage l (that is, EGC). Setting aside the cases of GC whose pathological stage could not be determined, the prevalence of cancers detected in the early stage was $86 \%$.

In conclusion, this prospective, long term, follow up study demonstrates the precancerous significance of non-invasive gastric neoplasia, its frequent association with $\mathrm{H}$ pylori infection, and the higher risk of progression to cancer associated with high grade lesions. Our results support both (i) the advisability of resective treatment (EMR or surgery) of any confirmed high grade non-invasive neoplasia and (ii) the biological rationale of following up low grade lesions. When lesions indefinite for non-invasive neoplasia coexist with $H$ pylori infection, eradication therapy and endoscopy follow up could result in a more suitable histological assessment of the lesion. Finally, our data suggest that an appropriate endoscopy follow up of non-invasive neoplasia increases the likelihood of GC being detected in its early, potentially curable, stage.

\section{ACKNOWLEDGEMENTS}

The IGGED participating units researchers include the following: Bassano-TV: B Crestani, A Guerini, P Scalon; Belluno: C Doglioni; Camposampiero e Cittadella-PD: C Angonese, C Chiavinato, E Lazzarini, L Ragni, S Saglimbeni, F Sonego; Castelfranco Veneto-TV: D Madia, G Mastropasqua, A Scapinello, E Venza; Chioggia-VE: E Dall'orso, C Sanavio; Dolo-VE: A D'Angelo, R Giordano, R Marin, A Montaguti; Feltre-BL: R Barbazza, R Cielo, M De Boni; Feltre-BL-Bellati: R De Bastiani, F Valiante; Padova: F Cardin, M Cassaro N Dal Bo, MC Fanton, F Foschia, F Farinati, M Guido, C Mescoli, R Naccarato, V Ninfo, G Pennelli, N Pennelli, M Rugge, VM Russo; Parma: F. Di Mario; VeneziaMestre: G Battaglia, A Capitanio, O Dal Maschio, B Murer, A Saggioro, V Stracca-Pansa; Vicenza: V Bertoncello, L Bozzola, A Campesato. This manuscript is dedicated to Rodger Haggitt. We remember him not only as one of the most prominent gastrointestinal pathologists of our time, but also as an unforgettable friend. This study was supported by the Roberto Farini Foundation for Research in Gastroenterology, by "CCC" (Cittadella contro il cancro) association, and by the Italian Minister for Scientific Research (MIUR) "Progetto Chiron Vaccino Helicobacter pylori-MIUR nr. Prat. 3133".

\section{Authors' affiliations}

M Rugge, M Cassaro, G Leo, V M Russo, G Pennelli, Department of Oncological and Surgical Sciences, III Cattedra di Anatomia Patologica, Università degli Studi di Padova-Azienda Ospedale Padova, Italia F Di Mario, Department of Gastroenterology, Università di Parma, Italia G Leandro, Department of Medicine, "Saverio de Bellis" Hospital, Castellana Grotte, Italia

F Farinati, Department of Surgical and Gastroenterology Sciences, Università degli Studi di Padova- Azienda Ospedale Padova, Italia

\section{REFERENCES}

1 Correa P. A human model of gastric carcinogenesis. Cancer Res 1988;48:3554-60

2 Correa P. Human gastric carcinogenesis: a multistep and multifactorial process. First American Cancer Society Award Lecture on Cancer Epidemiology and Prevention. Cancer Res 1992;52:6735-40.

3 Fenoglio-Preiser C, Carneiro F, Correa, et al. Gastric carcinoma. In: Hamilton SR, Aaltonen LA, eds. Pathology and Genetics, Tumors of the Digestive System. Lyon: IARC Press, 2000;39-52.

4 Morson BC, Sobin LH, Grundmann E, et al. Precancerous conditions and epithelial dysplasia in the stomach. J Clin Pathol 1980;33:711-21.

5 Jass JR. A classification of gastric dysplasia. Histopathology Jass JR. A classifica

6 Ming SC, Bajtai A, Correa P, et al. Gastric dysplasia. Significance and pathologic criteria. Cancer 1984;54:1794-801.

7 Tosi P, Baak JP, Luzi P, et al. Morphometric distinction of low- and high-grade dysplasias in gastric biopsies. Hum Pathol 1989:20:839-44.

8 Riddell RH. Premalignant and early malignant lesions in the gastrointestinal tract: definitions, terminology, and problems. Am J Gastroenterol 1996;91:864-72

9 Goldstein NS, Lewin KJ. Gastric epithelial dysplasia and adenoma: historical review and histological criteria for grading. Hum Pathol 1997; 28: 127-33

10 Lewin KJ. Nomenclature problems of gastrointestinal epithelial neoplasia. Am J Surg Pathol 1998;22:1043-7.

11 Riddell RH, Iwafuchi M. Problems arising from Eastern and Western classification systems for gastrointestinal dysplasia and carcinoma: are they resolvable? Histopathology 1998;33:197-202.

12 Schlemper RJ, Riddell RH, Kato Y, et al. The Vienna classification of gastrointestinal epithelial neoplasia. Gut 2000:47:251-5.

13 Takagi K, Sugano H, Nakamura K, et al. Clinicopathological study of atypical epithelial lesions of the stomach. Gan No Rinsho 1971;17:905-14

14 Rugge $M$, Cassaro M, Farinati F, et al. Diagnosis of gastric carcinoma in Japan and western countries. Lancet 1997;350:448.

15 Rugge M, Correa P, Dixon MF, et al. Gastric dysplasia: the Padova international classification. Am J Surg Pathol 2000 24:167-76.

16 Rugge M, Farinati F, Di Mario F, et al. Gastric epithelial dysplasia: a prospective multicenter follow-up study from the Interdisciplinary Group on Gastric Epithelial Dysplasia. Hum Pathol 1991;22:1002-8.

17 Rugge M, Farinati F, Baffa R, et al. Gastric epithelial dysplasia in the natural history of gastric cancer: a multicenter prospective follow-up study. Interdisciplinary Group on Gastric Epithelial Dysplasia. Gastroenterology 1994;107:1288-96.

18 Rugge M, Leandro G, Farinati F, et al. Gastric epithelial dysplasia. How clinicopathologic background relates to management. Cancer 1995;76:376-82

19 Riddell RH, Goldman H, Ransohoff DF, et al. Dysplasia in inflammatory bowel disease: standardized classification with provisional clinical applications. Hum Pathol 1983;14:931-68.

20 Rugge $M$, Cassaro M, Pennelli $G$, et al. Pathology and cost effectiveness of endoscopy surveillance for premalignant gastric lesions. Gut 2003;52:453-4.

21 UICC. Sobin LH, Witteking C, eds. TNM Classification of Malignant Tumors, 5th edn. New York: Wley-Liss, 1997

22 Rugge M, Correa P, Dixon MF, et al. Gastric mucosal atrophy: interobserver consistency using new criteria for classification and grading. Aliment Pharmacol Ther 2002;16:1249-59.

23 Neugut Al, Hayek M, Howe G. Epidemiology of gastric cancer. Semin Oncol 1996;23:281-91

24 Pagnini C, Rugge M. Advanced gastric cancer and prognosis. Virchows Arch A Pathol Anat Histopathol 1985;406:213-1.

25 Nishi $\mathbf{M}$, Ishihara S, Nakajima T, et al. Chronological changes of characteristics of early gastric cancer and therapy: experience in the characteristics of early gastric cancer and therapy: experience in the
Cancer Institute Hospital of Tokyo, 1950-1994. J Cancer Res Clin Oncol Cancer Institute Hosp

26 Weinstein WM, Goldstein NS. Gastric dysplasia and its management. Gastroenterology 1994;107:1543-5.

27 Rugge M, Cassaro M, Leo G, et al. Helicobacter pylori and gastric cancer: both primary and secondary preventive measures are required. Arch Intern Med 1999:159:2483-4.

28 Lauwers GY, Riddell RH. Gastric epithelial dysplasia. Gut 1999;45:784-90 
29 Parsonnet J, Axon AT. Principles of screening and surveillance. Am J Gastroenterol 1996:91:847-9.

30 Plummer $M$, Buiatti E, Lopez $G$, et al. Histological diagnosis of precancerous lesions of the stomach: a reliability study. Int J Epidemiol 1997; 26:716-20.

31 Farini R, Pagnini C, Farinati F, et al. Is mild gastric epithelial dysplasia an indication for follow-up? J Clin Gastroenterol 1983;5:307-10.

32 Saraga EP, Gardiol D, Costa J. Gastric dysplasia. A histological follow-up study. Am J Surg Pathol 1987;1 1:788-96.

33 Coma del Corral MJ, Pardo-Mindan FJ, Razquin S, et al. Risk of cance in patients with gastric dysplasia. Follow-up study of 67 patients. Cancer 1990;65:2078-85.

34 Koch HK, Oehlert M, Oehlert W. An evaluation of gastric dysplasia in the years 1986 and 1987. Pathol Res Pract 1990;186:80-4

35 Whiting JL, Sigurdsson A, Rowlands DC, et al. The long-term results of endoscopic surveillance of premalignant gastric lesions. Gut 2002;50:378-81
36 Fertitta AM, Comin U, Terruzzi V, et al. Clinical significance of gastric dysplasia: a multicenter follow-up study. Gastrointestinal Endoscopic Pathology Study Group. Endoscopy 1993;25:265-8.

37 Potet F, Camilleri JP. High risk populations and precancerous dysplasia in the stomach: definition and management. Gastroenterol Clin Biol 1982;6:454-61.

38 Kokkola A, Haapiainen R, Laxen F, et al. Risk of gastric carcinoma in patients with mucosal dysplasia associated with atrophic gastritis: a follow up study. J Clin Pathol 1996;49:979-84

39 Offerhaus GJ, Stadt J, Huibregtse K, et al. Endoscopic screening for malignancy in the gastric remnant: the clinical significance of dysplasia in gastric mucosa. J Clin Pathol 1984;37:748-54.

40 Lansdown M, Quirke P, Dixon MF, et al. High-grade dysplasia of the gastric mucosa: a marker for gastric carcinoma. Gut 1990;31:977-83.

1 Di Gregorio C Morandi P. Fante R et al Gastric dysplasia. A follow-up study. Am J Gastroenterol 1993:88:1714-19.

42 Gimenez Bascunana A, Bermejo Lopez J, Moreno Requena J, et al. An Gimenez Bascunana A, Bermejo Lopez J, Moreno Requena J, et al. An
evaluation of the clinical significance of gastric dysplasia: a histological follow-up study. Rev Esp Enferm Dig 1994;85:423-9.

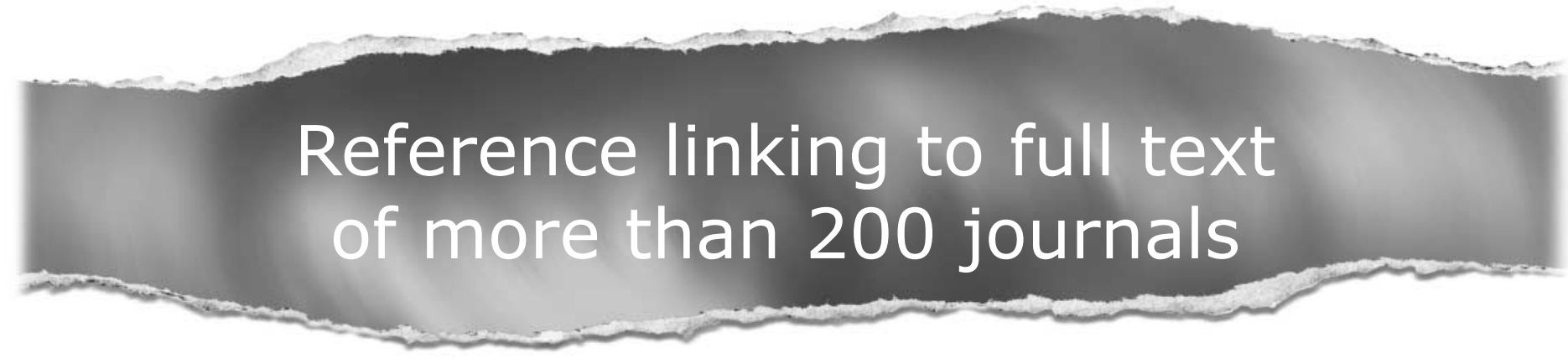

\section{Toll free links}

You can access the FULL TEXT of articles cited in Gut online if the citation is to one of the more than 200 journals hosted by HighWire (http://highwire.stanford.edu) without a subscription to that journal. There are also direct links from references to the Medline abstract for other titles.

\section{www.gutjnl.com}

